\title{
Magnetic Resonance Imaging of Human Brain Macrophage Infiltration
}

\author{
Klaus G. Petry, Claudine Boiziau, Vincent Dousset, and Bruno Brochet \\ University of Bordeaux, EA2966 Neurobiology of Myelin Diseases, Bordeaux, Cedex F-33076 France
}

\begin{abstract}
Summary: Macrophage tracking by magnetic resonance imaging (MRI) with iron oxide nanoparticles has been developed during the last decade for numerous diseases of the CNS. Experimental studies on animal models were confirmed by first clinical applications of MRI technology of brain macrophages for multiple sclerosis, ischemic stroke lesions, and tumors. As activated macrophages act in concert with other immune competent cells, this innovative MRI approach provides new functional data on the immune reaction in these CNS diseases. The MRI detection of brain macrophages defines precise spatial and temporal patterns of macrophage involvement that helps to characterize individual neurological disorders. This approach is being explored as an in vivo marker for the clinical diagnosis of cerebral lesion activity, in experimental models for the prog-
\end{abstract}

nosis of disease development, and to determine the efficacy of immunomodulatory treatments under clinical evaluation. Comparative brain imaging follow-up studies of blood-brain barrier leakage by MRI with gadolinium-chelates, microglia activation by positron emission tomography with radiotracer ligand PK11195 and MRI detection of macrophage infiltration provide more precise information about the pathophysiological cascade of inflammatory events in cerebral diseases. Such multimodal characterization of the inflammatory events should help in the monitoring of patients, in defining precise time intervals for therapeutic interventions, and in developing and evaluating new therapeutic strategies. Key Words: Macrophages, MRI, brain diseases, multiple sclerosis, stroke, glioma, iron oxide nanoparticles.
Activated macrophages, in concert with other immune competent cells, play a critical role in determining immunogenicity and the generation of appropriate immune responses. Under healthy physiological conditions, immune cell migration into the CNS is very restricted. In contrast, during many pathophysiological processes of the CNS, including inflammation and autoimmune diseases, immune competent cells, and macrophages, cross the blood CNS barriers (blood-brain barrier [BBB] and blood-CSF barrier) to infiltrate the CNS parenchyma.

In vivo monitoring of the pathophysiological cascade of events of immune cell migration into the CNS represents a major opportunity as it could generate in vivo markers for both diagnosis and prediction of disease development. In addition, such capabilities could open new perspectives for potential therapeutic targets and provide a novel means to evaluate the efficacy of therapeutic strategies. To characterize the extravasation of

Address correspondence and reprint requests to: Klaus G. Petry, Ph.D., University of Bordeaux 2, EA2966 Neurobiology of Myelin Diseases Laboratory, 146, rue Léo-Saignat/BP78, Bordeaux, Cedex F-33076 France. E-mail: klaus.petry@ bordeaux.inserm.fr. immune cells from the vascular space into the brain parenchyma, technical approaches using genetically engineered fluorescent cells, and the specific labeling with dyes and contrast agents, provide a means to observe the migration of cells in vivo.

Activated macrophages have complex biologic functions and characteristics that have been well documented in recent years. ${ }^{1}$ The inflammatory profile of monocytes and macrophages is complex and may control the development of T helper (Th1/Th2) adaptive immunity. Activation and recruitment of blood monocytes to the inflammatory sites provides an important source of tissue macrophages. Macrophages are highly versatile phagocytic cells whose diverse effector functions can selectively be programmed by an array of environmental signals. ${ }^{2}$ To date, a current concept has emerged categorizing macrophages into at least two distinct phenotypic subsets i.e., classically (M1) or alternatively (M2) activated macrophages. ${ }^{3}$ Priming with interferon- $\gamma$, tumor necrosis factor- $\alpha$ or lypopolysaccaride (LPS) will program macrophages into the M1 phenotypic state, which is distinguished by a strong proinflammatory response and potent bacterial killing. In contrast, exposure 
to interleukin-4 or interleukin-13 generates M2 immunophenotype macrophages, which display an attenuated proinflammatory and enhanced anti-inflammatory/immunomodulatory response. Based on these effector differences, it has been hypothesized that the proinflammatory cytokine pattern produced by the M1 subset of macrophages is conducive to the development of Th1type adaptive immunity, whereas the anti-inflammatory cytokine pattern generated by the M2 subset of macrophages corresponds with a Th2 type of immune response.

The capacity to phagocyte particles and compounds and to migrate to sites of inflammation has provided the rationale for in vivo labeling and monitoring of macrophages. Upon intravenous injection, coated superparamagnetic iron oxide (SPIO) nanoparticles are captured by the monocyte-macrophage system. Although these nanoparticles are not completely cleared from the circulating bloodstream by the liver and spleen, they are captured by the professional phagocytes in the blood circulation. The endocytotic loading of the circulating macrophages with nano-technology-based contrast agents containing iron oxide was originally developed to monitor lymphomas in vivo by MRI. ${ }^{4}$ In recent years, this intriguing approach has initiated the development of novel MRI techniques for the noninvasive observation of inflammatory and degenerative disorders involving strong phagocytic activity of macrophages by using iron oxide nanoparticles as contrast agents. ${ }^{5,6}$

Experimental data have shown that the size of the iron oxide nanoparticles when injected intravenously is critical for clearance from the blood circulation. The hydrodynamic particle size for coated SPIO is between 60 and $150 \mathrm{~nm}$ diameter for Ferumoxides (Endorem), and Ferucarbotran (Resovist), and for dextran-coated ultrasmall superparamagnetic iron oxide (USPIO) nanoparticles between 10 and $40 \mathrm{~nm}$ diameter for Ferumoxtran-10 (Combidex, Sinerem). These sizes imply specific biological features in the interaction with professional phagocytes. After intravenous administration, SPIO nanoparticles are rapidly cleared from the blood circulation by cells of the reticuloendothelial system in the spleen and liver, making them more adaptable for imaging for these tissues (i.e., liver tumors). However, SPIO nanoparticles are also captured by circulating phagocytes in the blood. Circulation times of USPIO nanoparticles are considerably longer with a blood circulation half-time in rats of approximately $5 \mathrm{~h}$ and in humans of 24 to $36 \mathrm{~h}$, which may favor their uptake by phagocyte populations in lymph nodes, ${ }^{4}$ but also by phagocytes in the circulating blood. The uptake of SPIO and USPIO nanoparticles by the professional phagocytic monocytes and macrophages in the circulating blood provides a tool of choice to monitor the involvement of macrophages in inflammatory processes of CNS diseases, such as multiple sclerosis, traumatic nerve injury, cerebral stoke, and brain tumors in vivo, by MRI, in experimental and clinical investigations. In these diseases, macrophage imaging has opened new applications for diagnosis, but also for the prediction of disease development and evaluation of therapeutic strategies.

\section{MULTIPLE SCLEROSIS}

Multiple sclerosis (MS) is characterized by the formation of disseminated inflammatory lesions (plaques) throughout the CNS, mainly in the brain and spinal cord white matter, but also in the cortex. These lesions have features of axonal and neuronal loss, added to demyelination, which are considered the main causes of the progressive physical and cognitive handicap. The MS handicap develops during inflammatory relapses that are characterized by resident microglial cell activation in the CNS (see Cagnin et al. ${ }^{7}$ ) and penetration of circulating T and B lymphocytes, neutrophils, and macrophages from the blood compartment into the CNS parenchyma due to various degrees of BBB permeability. The precise causes of MS remain unknown because of the complexity of interacting factors implicated in the loss of immune homeostasis, myelin, and axonal injury, to finally generate progressive neurological impairments. ${ }^{8}$

The interactions and interdependencies among inflammation, demyelination, neurodegeneration, and neuroprotection during the pathological process, or during the relapsing remitting MS disease progress are still poorly understood. Magnetic resonance imaging has largely contributed to advances in monitoring lesions by detecting the increased leakage of the BBB using gadoliniumchelate (Gd) contrast agents, and in determining the demyelinating and remyelinating process by using magnetization transfer imaging sequences to appreciate the interactions between water molecules and macromolecular structures, in particular membranes (see Filippi et al. ${ }^{9}$ ). The study of different experimental autoimmune encephalomyelitis (EAE) models and a toxic demyelinating model of the corpus callosum demonstrated (during a process of demyelination followed by remyelination) the strong correlation between the alterations determined in vivo by magnetization transfer and the degree of the structural affection in histology and immunohistochemistry. ${ }^{10}$ Our data largely contributed to the evaluation of this technique for in vivo studies of MS lesion profiles ${ }^{11}$ and of remyelination in MS, which may occur spontaneously or after treatment. Although these MRI parameters are helpful in evaluating the consequences of the CNS inflammation, they do not provide information on the cellular inflammatory process itself. Furthermore, these MRI parameters have only minor predictive value for formation of plaques and for the severity of relapses that are at the origin of the MS disease aggravation. Moreover, the identification of early 
markers of disease activity is needed for the assessment of therapeutic strategies.

For more than a decade, the proinflammatory role of macrophages in the inflammatory process of MS has been described. ${ }^{12}$ However, macrophages play various functions in MS, and the more recent distinction in proinflammatory M1 and immunomodulatory M2 phenotypes of macrophages is not characterized during MS disease development.

\section{CNS INFILTRATION OF MACROPHAGES IN RELATION TO BBB}

The transgression of the BBB by inflammatory cells within the interplay between microglia cells and bloodborne macrophages in the inflammatory process of the CNS is still poorly understood. The application of MRI with iron oxide nanoparticles in EAE animal models of MS and the transfer of this technological innovation in a first MS clinical study have provided new in vivo insights in the cellular inflammatory process.

The assessment of macrophage CNS infiltration by MRI with USPIO, in close comparison with leakage of the BBB as evidenced by MRI with Gd, clearly distinguished both events. Depending on the EAE animal models and on the disease stage of MRI analysis, it seems that Gd-enhanced BBB leakage and brain infiltration by USPIO-loaded macrophages are not exactly concomitant. Indeed, in acute EAE, Dousset et al. ${ }^{13}$ and Rausch et al. ${ }^{14}$ detected lesions either with USPIO as a marker for macrophage infiltration or Gd as a marker for BBB leakage alone; some lesions were detected with both contrast agents. In all these experiments, one should recall that Gd is injected shortly before performing MRI scans, whereas USPIO is injected $24 \mathrm{~h}$ before MRI evaluation to obtain maximal MRI signal alterations. Therefore, the time of macrophage infiltration cannot be precisely determined. In an elegantly performed kinetic analysis, Floris et al. ${ }^{15}$ observed dramatic MRI signal changes with USPIO within 2 days. In this study, macrophage infiltration unambiguously occurred when the first clinical symptoms scored as flaccid tail were already detected.

One major point needs to be considered in the MRI timing and evaluation of lesion formation involving both Gd-enhanced BBB rupture and USPIO-enhanced macrophage ingress. For technical reasons, to avoid breathing artifacts, spinal cord MRI is rarely performed in experimental studies. The MRI studies mainly evidenced lesions in the brain and upper spinal cord at the first visible, clinically-based, motor function scoring. However, EAE and its severity is immunologically already set up at the early, even preclinical, disease phase. ${ }^{16} \mathrm{We}$ have further evidence that EAE is initiated and in progress during the preclinical phase causing alterations that are clinically not yet visible (i.e., bladder dysfunction). ${ }^{17}$ Thus many alterations, including macrophage ingress and BBB leakage, are already in progress, at least in the spinal cord, but are not yet detectable by MRI in the preclinical stage. ${ }^{18}$

In all of these EAE studies, histological examinations confirmed that iron oxide nanoparticles were mainly localized in macrophages in the CNS parenchyma, but also to a minor extent in endothelial cells. The question arose whether the MRI signal alteration was indeed reflecting active transport of iron oxide nanoparticles by infiltrating macrophages after uptake in the circulating blood or whether the uptake by CNS macrophages/microglia of nanoparticles after transcytosis at the BBB is the main route of their accumulation in the CNS. The elegantly performed comparison of peripheral nerve injury involving macrophage infiltration and microglia activation with optic nerve injury provided conclusive evidence that the accumulation of iron oxide nanoparticles is dependent on the uptake by macrophages in the circulating blood, and the active transport by these cells into the nervous tissue. $^{19}$

A first human application of the macrophage imaging approach by MRI in MS was performed in patients with definite relapsing remitting MS. ${ }^{20}$ During acute relapse, 10 MS patients were monitored with Gd and USPIO (Sinerem). At the first MRI, Gd was administered and seven of these patients showed MS lesion enhancement with Gd. Then, USPIO was administered and the patients were monitored by MRI at days 1 and 3 after administration. At day 1, 9 of 10 patients showed MS brain lesion enhancement with USPIO. Among a total of 57 MRI-enhanced lesions, three patterns were distinguished when comparing Gd-related and USPIO-related enhancement: 1) the lesions enhanced with both contrast agents $(\mathrm{n}=31), 2)$ with Gd only $(\mathrm{n}=24)$, and 3$)$ with USPIO only $(\mathrm{n}=2)$ (FIG. 1).

These results obtained with MS patients are in agreement with previous observations in EAE. Some MS lesions enhanced for up to 3 days after USPIO administration (Dousset et al., unpublished observations). A heterogeneous population study including some MS patients imaged by MRI with ferumoxtran-10 compared to $\mathrm{Gd}$ revealed heterogeneous enhancement patterns with little or no USPIO uptake. ${ }^{21}$

Considering that involvement of macrophages contributes to the heterogeneity of MS lesions and disease development, ${ }^{22}$ what does this new in vivo characterization of MS lesions by MRI with USPIO and Gd imply with respect to the pathological and clinical findings of MS?

MRI detection of macrophage CNS infiltration seems to be, at least to a certain degree, spatially and temporally independent from the BBB permeability. The very dynamically evolving inflammatory process that is characterized by the passage of the BBB by immune competent 
Gd
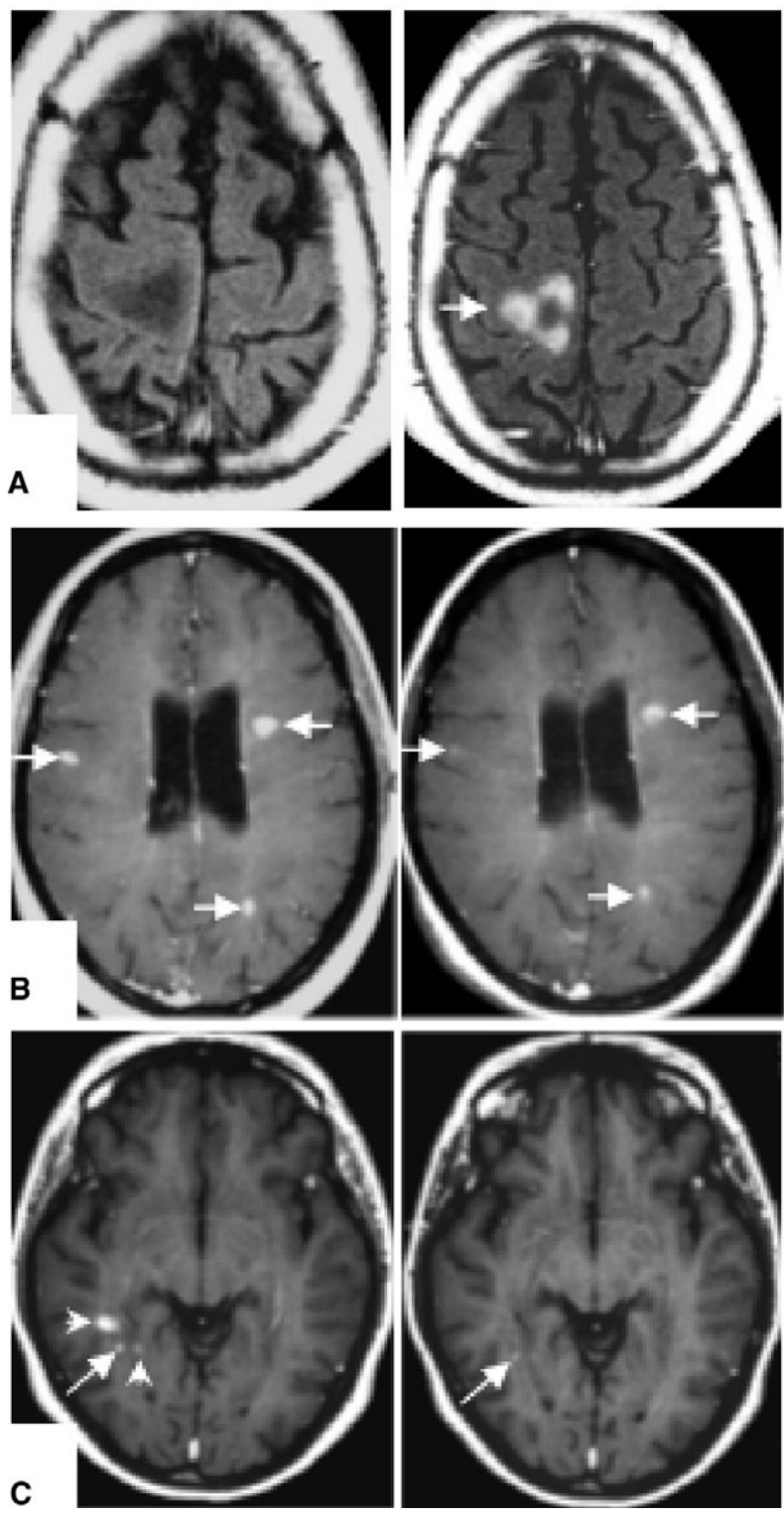

FIG. 1. Magnetic resonance imaging patterns of macrophage brain infiltration with ultrasmall superparamagnetic iron oxide particles (USPIO) (left panels) and blood-brain barrier (BBB) leakage with gadolinium-chelate (Gd) (right panels) in acute multiple sclerosis. Pattern A shows a large lesion that was unenhanced by Gd on T1-weighted images (T1WI) indicating no BBB leakage, but contains high-signal intensity on T1WI with USPIO reflecting massive macrophage infiltration. Pattern $\mathbf{B}$ shows congruent lesion formation with both BBB leakage (Gd enhancement) and macrophage infiltration (USPIO enhancement) on T1WI. Pattern C shows T1WI of Gd-enhanced BBB leakage in three lesions of which only one is enhanced with USPIO on T1WI. Arrows indicate enhanced lesions. (Partially reproduced from Dousset et al. ${ }^{18}$ with permission.)

cells, including macrophages and the BBB leakage, should be considered in the interpretation of the data. Indeed, the accumulation of USPIO in CNS can be considered as an in vivo marker for infiltrating macrophages that is mainly associated with, but distinct from, BBB rupture.

On the other hand, the observation of Gd-enhancing lesions that are negative for USPIO macrophage infiltration might reveal only BBB leakage without infiltrating phagocytic cells. Although the precise significance of $\mathrm{Gd}$ enhancement remains unknown, the discrepancy with USPIO-enhancing lesions indicates that various pathophysiological mechanisms are contributing to disease activity and long-term disability in patients. However, whether these Gd-enhancing lesions are in an immunological active status, as activated microglia, without involvement of infiltrating immune cells of the circulating blood, remains a question.

The presence of myelin degradation products in macrophages is considered a criterion of MS inflammatory lesion activity. In MS lesions, however, myelin ingestion induces a foamy appearance of myeloid cells and confers an anti-inflammatory function as these cells lack proinflammatory cytokines and express anti-inflammatory molecules. ${ }^{23}$ Furthermore, in relapsing EAE, activation patterns of perivascular macrophages within the inflammatory CNS lesions presenting the proinflammatory (M1) and immunomodulatory (M2) characteristics, indicate a heterogeneity of the macrophage population. Quantitatively, the M1/M2 balance of macrophages in the CNS varies with relapsing EAE disease development. ${ }^{24}$ Furthermore, although after severe relapsing EAE, when animals have completely recovered from their clinically visible motor impairments, the often observed continuing macrophage infiltration of the CNS as monitored by MRI with USPIO indicates characteristics other than the proinflammatory macrophage phenotype, which is seen in acute disease and relapses (Mikita et al., unpublished data). Magnetic resonance imaging detection of single cells in the brain ${ }^{25}$ permits the perspective to better characterize individual infiltrating macrophages in CNS parenchyma with respect to their pathophysiological immune response function (see Slotkin et al. ${ }^{26}$ for more information on single cell MRI).

\section{RELATIONSHIP BETWEEN T CELL CNS INFILTRATION AND MACROPHAGES}

MS is mostly described as a Th1 lymphocytic disease. ${ }^{27}$ Therefore, understanding the association between macrophage activation, $\mathrm{T}$ lymphocyte phenotype, and inflammatory attacks is of great interest. The cellular composition of inflammatory lesions, together with the local production of various compounds associated with inflammation, suggests that the cascade of inflammatory events is mediated by T-cell infiltration. In response, an alteration of the BBB allows the recruitment of other inflammatory cells, including macrophages. In the CNS, autoreactive $\mathrm{T}$ cells and macrophages exert their destruc- 
tive activity and are finally responsible for myelin and axon loss. The various infiltration steps represent potential targets to block inflammation. Inhibition of T-cell infiltration by antibodies reactive with the VLA4 antigen, which inhibit the anchoring of $\mathrm{T}$ cells to endothelial cells at the BBB, is a promising therapeutic approach for MS. After treatment of an initiated EAE in rats with antiVLA4 antibodies (TA2/Natalizumab; Biogen, Cambridge, Mass), a residual CNS infiltration of macrophages can be detected by MRI with USPIO, despite the total suppression of infiltrating $\mathrm{T}$ cells. ${ }^{28}$ These data reveal dissociation between activation of $\mathrm{T}$ cells and macrophages. In the initiated but interrupted inflammation process, the macrophage infiltration may persist in the absence of T-cell infiltration.

\section{MACROPHAGE MRI UNDER IMMUNOMODULATORY TREATMENT}

Magnetic resonance imaging with USPIO has shown the ability to monitor macrophage CNS infiltration in the autoimmune process. This approach has opened new perspectives to validate the use of iron oxide nanoparticles as a noninvasive tool to evaluate the efficacy of therapeutic strategies, qualitatively, and quantitatively.

Natalizumab, intravenously administered, inhibits the interaction of $\alpha-4$ integrins expressed on leukocytes with their respective endothelial counter receptor vascular cell-adhesion molecule, thus preventing leukocytes from crossing the BBB. ${ }^{29}$ Although the EAE treatment study with anti-VLA4 antibodies (TA2/natalizumab) revealed persisting macrophage ingress by MRI with USPIO, and despite the complete suppression of T-cell infiltration in an ongoing inflammatory disease process, the sizes of lesions detected by USPIO and MRI were significantly reduced. ${ }^{28}$ The data evidenced an immediate effect of this therapeutic strategy that can be quantitatively measured in vivo by MRI detection of USPIO-loaded macrophages. The MRI data correlated with histologically determined perivascular macrophage infiltration. ${ }^{28}$

An orally administered ligand of sphingosine-1 phosphate receptors (FTY720) significantly reduced the frequency of new Gd-enhanced lesions on MR and clinical relapses in patients with relapsing-remitting MS. ${ }^{30}$ This compound deprives lymphocytes of expression of the receptor necessary to egress from secondary lymphoid tissues. Lymphocytes are thus sequestered in lymph nodes. In acute and relapsing stages of EAE, daily oral administration of FTY720 suppressed clinical disease development and neurological impairment. The MRIbased in vivo tracking of USPIO-labeled macrophages correlated with histological and clinical EAE evaluation. $^{31}$

Statins are largely used to lower cholesterol levels. The immunomodulatory activity of statins has been found to reduce the formation of Gd-enhancing lesions in an open-labeled study of patients with relapsing-remitting MS. ${ }^{32}$ The immunomodulator lovastatin suppresses the activity of 3-hydroxy-3-methylglutaryl coenzyme A reductase. The EAE animals were treated with lovastatin, which ameliorated the clinical outcome. The MRI showed that the USPIO load in the brain was significantly diminished in lovastatin-treated animals. ${ }^{13}$

In the pertussis toxin CNS-inflammatory model applied to CCL2 overexpressing transgenic mice, leukocytes accumulate spontaneously in the perivascular space in brains but do not cross the glia limitans. The USPIOMRI and immunological characterization identified leukocyte migration across the glia limitans, into the brain parenchyma that involves metalloproteinase enzymes, as the critical step in inducing clinical symptoms. Weight loss and parenchymal infiltration, but not perivascular accumulation of leukocytes, were significantly inhibited by the broad-spectrum metalloproteinase inhibitor BB94/Batimastat. ${ }^{33}$

Conclusively, in all of these therapeutic EAE studies, in vivo MRI tracking of macrophages with USPIO correlated with the evolution of clinical disease and the histological findings under therapeutic intervention. In vivo MRI monitoring of macrophages in EAE provides direct evidence of the immunomodulatory efficacy of therapeutics actually under clinical evaluation. These studies emphasize that the in vivo macrophage cell tracking with USPIO is a valuable tool to quantitatively assess the efficacy of new therapeutics in preclinical and potentially clinical studies. On the other hand, the remaining macrophage infiltration, despite the efficacy of the treatment, might reflect the immunomodulatory action of macrophages.

\section{FOLLOW-UP MRI OF MACROPHAGES AND PREDICTION OF DISEASE SEVERITY}

The MRI follow-up during relapsing EAE indicates that the uptake of USPIO decreases during relapses. ${ }^{34}$ This observation may be explained by a less severe, second clinical attack often observed in relapsing EAE. In the relapsing EAE in the Dark Agouti rat, individual MRI follow-up for macrophage CNS infiltration allows the discrimination at clinical disease onset between animals with or without macrophage ingress into the CNS. Independently of positive or negative MRI for macrophage ingress, the EAE animals showed no differences in severity of the first clinical attack. However, only the macrophage MRI positive rats developed a relapsing disease process with more important demyelination and axonal suffering. Furthermore, axonal loss related to peroxynitrite production was only observed in animals with repeated clinical attacks. These data document that the inter-individual differences of macrophage MRI at onset 
of the acute EAE phase has a predictive value for clinical outcome. ${ }^{35}$ Furthermore, some animals in the relapsing EAE rat model with a positive MRI for macrophage infiltration at disease onset developed the most severe disease with a first relapse for up to 12 days including complete paralysis (clinical score, 4) and repeated relapses. In these animals, the macrophage infiltration at the first MRI is mainly seen in the upper spinal cord and brain stem, and spreads into various parts of the CNS with disease development (Petry et al., unpublished data). All together, these observations confirm that MRI with USPIO reflects the infiltration of activated macrophages into the CNS. The inter-individual variation of sensibility to the disease is, as for humans, not precisely understood, and mechanisms causing the relapses in the animal models are not explained.

\section{CEREBRAL ISCHEMIA}

Cerebral ischemic strokes induce a cascade of focal inflammatory phagocytic cell activation that is linked to the occurring apoptotic cell death. Microglia cells respond immediately at stroke onset by the release of inflammatory compounds. Activated microglia cells can be detected in vivo by positron emission tomography (PET) with PK11195, a radioactive ligand to peripheral-type benzodiazepine-binding sites on mononuclear phagocytes (see Banati et al. ${ }^{7}$ ). They cause exacerbation of tissue damage, but they may also protect the brain against excitotoxicity. ${ }^{36,37}$ Histologically, it has been documented that blood-borne macrophages infiltrate the ischemic area with a delay of at least $24 \mathrm{~h}$ and with a main infiltration between 3 and 6 days. ${ }^{38,39}$

Different experimental ischemia animal models were studied in vivo by MRI to determine the infiltration of hematogenous macrophages in the infarctions with iron oxide nanoparticles. The obtained MRI data were confirmed histologically for macrophage infiltration. In experimental permanent or transient middle cerebral artery occlusion ischemia models, in vivo macrophage monitoring by MRI with intravenously-administered USPIO (ferumoxtran-10) confirmed the delayed infiltration into the ischemic area. After ischemia induction (serial MRI) for the next 7 days revealed increasing signal alterations in the ischemic zone with a peak of signal alterations on T2-weighted images at day $4 .^{40,41}$ Photochemically-induced permanent occlusion of cortical microvessels revealed accumulating SPIO-enhanced MRI signal alterations at the border of infarctions during the early stages of lesion development, ${ }^{42,43}$ which were mostly detected at days 5 and 6 after ischemia induction. ${ }^{44}$ The MRIUSPIO study of Wiart et al. ${ }^{45}$ adds new information on the spatial dynamics of in vivo phagocyte labeling in murine focal brain ischemia. The USPIO-enhanced MRI kinetic analysis disclosed an inflammatory response sur- rounding the ischemic lesion and in the contralateral hemisphere via the corpus callosum to the whole cortex and striatum, and suggested a spread of USPIO-related signal from the ipsilateral to the contralateral hemisphere. The MRI data correlated with histochemical analysis showing inflammation remote from the lesion and ingestion of nanoparticles by microglia/macrophages.

Saleh et al. ${ }^{46}$ performed the first clinical MRI study of ischemic stroke in 10 patients comparing Gd and USPIO contrast agents. Corresponding to the defined time delay of macrophage CNS infiltration in experimental studies, MRI scans were performed 4 to 6 days after stroke onset. After the evaluation of Gd MRI enhancement, which was observed in 3 patients, USPIO was administered intravenously and MRI scans performed $24 \mathrm{~h}$ and $48 \mathrm{~h}$ later revealed signal alterations in all 10 patients. Different USPIO-related signal changes were observed. Although blood vessel-associated signal alterations in T2-weighted images decreased from the first to second MRI after USPIO infusion, the mainly observed USPIO parenchymal enhancement on T1-weighted images increased with time. The MRI signal decreases are explained by the clearance of USPIO from the blood circulation (half time, $24 \mathrm{~h}$ to $36 \mathrm{~h}$ ). The cerebral MRI signal correlated with the expected distribution of macrophages and reflects the infiltration of macrophages from the peripheral blood. A similar clinical study ${ }^{47}$ on day 9 revealed heterogeneous MRI signal alterations after USPIO injection in 9 of 10 stroke patients on T1-weighted images, and in 5 of 10 patients on $\mathrm{T} 2 *$-weighted images. Lesion volume on day 6 on diffusion-weighted images had no relationship to USPIO enhancement at day 9. No relationship between BBB leakage (detected by Gd enhancement) and USPIO enhancement was observed. Furthermore, MRI detection of macrophage infiltration was heterogeneous and not clearly related to subacute lesion volume.

The experimental and clinical data obtained in cerebral ischemia conclusively shows that MRI detection of macrophage activity with iron oxide nanoparticles provides different information than the Gd-associated rupture of the BBB. Furthermore, MRI detection of macrophage activity reveals new information on the spatiotemporal activation of phagocytic cell types associated with the inflammatory response for several days. Compared with the immediate microglia activation, as shown with PET (PK11195) studies (see Banati et al. ${ }^{7}$ ), infiltration of macrophages occurs at later stages (several days after infarction). The appropriate timing of contrast agent injection and subsequent MRI is therefore most critical for iron oxide nanoparticle-based macrophage imaging in brain ischemia. At these later stages of brain ischemia, the protection of CNS tissue is already initiated by suppressing proinflammatory compounds in favor of expression of anti-inflammatory factors. ${ }^{48}$ Spreading of 
microglia activation beyond the primary local infarction lesion site to the nonischemic brain areas has been documented in animal stroke models ${ }^{49}$ and in PET follow-up of stroke patients. ${ }^{50}$ Microglia activation in these, primarily unaffected brain areas, appears as the preceding and predominating event before the recruitment of blood borne macrophages within these areas. ${ }^{38,39}$ Such successive activation outside of the local lesion that also involves macrophages can be shown by MRI with USPIO. ${ }^{45}$ The distinct temporal and spatial characteristics of phagocyte activation opens new questions concerning their role in the pathophysiological processes of cerebral ischemia.

\section{GLIOMAS}

The SPIO and USPIO nanoparticles have been shown to accumulate both in experimental ${ }^{51}$ and human gliomas indicating phagocytic cells as potential therapeutic targets. The perfusion heterogeneity of tumor microvasculature proliferation and abnormalities of functional blood vessels induce the difficulty in determining the distribution and uptake of nanoparticles. The physicochemical properties of USPIO nanoparticles imply a prolonged blood circulation before uptake by tissue macrophages. These dual characteristics allow the MRI visualization of tumor-associated microvessels because USPIO nanoparticles circulate in the blood, and do not cross the intact BBB during the early period after intravenous administration, and after their clearance from the vascular compartment, the characterization of tumor-tissue associated macrophage activity.

Neuwelt et al. ${ }^{52}$ conducted the first clinical trials with MRI monitoring of macrophages in brain tumors. In 20 patients ${ }^{53}$ and 7 patients, ${ }^{52}$ respectively, with primary and metastatic intracranial tumors, comparing SPIO (ferumoxides) and USPIO (ferumoxtran-10), the investigators showed that most patients given USPIO had MRI-signal alterations reflecting the presence of iron oxide particles in the tumor. The USPIO was observed at $6 \mathrm{~h}$ post-infusion in the vascular compartment of brain tumors. In contrast, $24 \mathrm{~h}$ postinfusion, USPIO was found for up to 5 to 7 days, mainly in the margin, with less central localization of the brain tumors. Histological examination confirmed the iron uptake in parenchymal cells that represented macrophages and reactive astrocytes, not tumor cells. The interesting histological characterization of USPIO uptake in brain tumors raises the question of how was the intravenous-administered USPIO incorporated by activated astrocytes? A study of 55 patients ${ }^{54}$ with brain tumors in the glial tumor group revealed the uptake of USPIO in 19 of 34 cases. A study performed by Taschner et al. ${ }^{55}$ revealed USPIO-related MRI signal changes in 7 of 9 patients with brain tumors
$24 \mathrm{~h}$ after USPIO infusion. Iron histochemistry confirmed the presence of iron deposits in macrophages.

The MRI detection of macrophages in brain tumors depends on the time delay after USPIO infusion. The MRI interpretation with USPIO provides a variety of data. Late tumor MRI with USPIO reflects macrophage activity associated with brain tumors, which was more important in high-grade than low-grade glioblastomas. ${ }^{5}$ In the operative management of gliomas, the incorporation of iron oxide nanoparticles by macrophages in vivo that colocalize with brain tumors offers complementary information to the Gd-enhanced MRI detection of BBB leakage, and may also help to differentiate between brain tumors and areas of radiation necrosis. The macrophage MRI detection with USPIO of tumor morphology may help in surgical resection or biopsy of brain tumors.

\section{CONCLUSIONS}

Macrophage tracking by MRI with iron oxide nanoparticles provides new functional data on the immunologic reaction within the CNS in various disease states. The human MRI macrophage studies with SPIO or USPIO show no significant side effects related to injection of these agents. The reasons for the distinct USPIOrelated signal patterns on T1-weighted versus $\mathrm{T} 2{ }^{*}$ weighted images are currently unknown. They might correspond to the local concentration of USPIO, eventually reflecting the quantitative accumulation of macrophages. The experimental data confirmed by the first human applications of brain MRI macrophage detection in a variety of neurological disorders, such as MS, ischemic strokes, and tumors, suggest a spatial and temporal pattern of macrophage involvement for each of the CNS diseases. Comparative longitudinal imaging studies of microglia activation, BBB rupture, and macrophage infiltration will give more precise information on the status of brain inflammation. The new characterization of the cascade of inflammatory events will help in monitoring patients and may help define new therapeutic targets, determine appropriate time intervals for therapeutic interventions, and evaluate the efficacy of treatments.

Acknowledgments: The work was supported by Conseil Regional d'Aquitaine, ANR-Technologie pour la Santé 2006-40 NanoBioImaging and INSERM. We acknowledge the interactive collaboration with the Guerbet group and the generous gift of Sinerem for our MRI studies.

\section{REFERENCES}

1. Mosser DM. The many faces of macrophage activation. J Leuk Biol 2003;73:209-212.

2. Stout RD, Jiang C, Matta B, Tietzel I, Watkins SK, Suttles J. Macrophages sequentially change their functional phenotype in response to changes in microenvironmental influences. J Immunol 2005;175:342-349. 
3. Mantovani A, Sica A, Sozzani S, Allavena P, Vecchi A, Locati M. The chemokine system in diverse forms of macrophage activation and polarization. Trends Immunol 2004;25:677-686.

4. Weissleder R, Elizondo G, Wittenberg J, Lee AS, Josephson L, Brady TJ. Ultrasmall superparamagnetic iron oxide: an intravenous contrast agent for assessing lymph nodes with MR imaging. Radiology 1990;175:494-498.

5. Corot C, Petry KG, Trivedi R, et al. Macrophage imaging in central nervous system and in carotid atherosclerotic plaque using ultrasmall superparamagnetic iron oxide in magnetic resonance imaging. Invest Radiol 2004;39:619-625.

6. Corot C, Robert P, Idee JM, Port M. Recent advances in iron oxide nanocrystal technology for medical imaging. Adv Drug Deliv Rev 2006;58:1471-1504.

7. Cagnin A, Kassiou M, Meikle SR, Banati RB. Positron emission tomography imaging of neuroinflammation. Neurotherapeutics 2007;4:443-452.

8. Hauser SL, Oksenberg JR. The neurobiology of multiple sclerosis: genes, inflammation, and neurodegeneration. Neuron 2006;52:6176.

9. Filippi M, Rocca MA. Magnetization transfer magnetic resonance imaging of the brain, spinal cord, and optic nerve. Neurotherapeutics 2007;4:401-413.

10. Deloire-Grassin MS, Brochet B, Quesson B, et al. In vivo evaluation of remyelination in rat brain by magnetization transfer imaging. J Neurol Sci 2000;178:10-16.

11. Brochet B, Dousset V. Pathological correlates of magnetization transfer imaging abnormalities in animal models and humans with multiple sclerosis. Neurology 1999;53(suppl 3):S12-S17.

12. Bauer J, Ruuls SR, Huitinga I, Dijkstra CD. The role of macrophage subpopulations in autoimmune disease of the central nervous system. Histochem J 1996;28:83-97.

13. Dousset V, Ballarino L, Delalande C et al. Comparison of ultrasmall particles of iron oxide (USPIO)-enhanced T2-weighted, conventional T2-weighted, and gadolinium-enhanced T1-weighted MR images in rats with experimental autoimmune encephalomyelitis. AJNR Am J Neuroradiol 1999;20:223-227.

14. Rausch M, Hiestand P, Baumann D, Cannet C, Rudin M. MRIbased monitoring of inflammation and tissue damage in acute and chronic relapsing EAE. Magn Reson Med 2003;50:309-314.

15. Floris S, Blezer EL, Schreibelt G, et al. Blood-brain barrier permeability and monocyte infiltration in experimental allergic encephalomyelitis: a quantitative MRI study. Brain 2004;127(Pt 3): 616-627.

16. Boullerne AI, Rodriguez JJ, Touil T, et al. Anti-S-nitrosocysteine antibodies are a predictive marker for demyelination in experimental autoimmune encephalomyelitis: implications for multiple sclerosis. J Neurosci 2002;22:123-132.

17. Vignes JR, Deloire MS, Petry KG, Nagy F. Characterization and restoration of altered inhibitory and excitatory control of micturition reflex in experimental autoimmune encephalomyelitis in rats. J Physiol (Lond.) 2007:578(Pt 2):439-450.

18. Dousset V, Gomez C, Petry KG, Delalande C, Caille JM. Dose and scanning delay using USPIO for central nervous system macrophage imaging. MAGMA 1999;8:185-189.

19. Bendszus M, Stoll G. Caught in the act: in vivo mapping of macrophage infiltration in nerve injury by magnetic resonance imaging. J Neurosci 2003;23:10892-10896.

20. Dousset V, Brochet B, Deloire MS, et al. MR imaging of relapsing multiple sclerosis patients using ultra-small-particle iron oxide and compared with gadolinium. AJNR Am J Neuroradiol 2006;27: $1000-1005$.

21. Manninger SP, Muldoon LL, Nesbit G, Murillo T, Jacobs PM, Neuwelt EA. An exploratory study of ferumoxtran-10 nanoparticles as a blood-brain barrier imaging agent targeting phagocytic cells in CNS inflammatory lesions. ANJR Am J Neuroradiol 2005; 26:2290-2300.

22. Lassmann H, Brück W, Luchinetti CF. Heterogeneity of multiple sclerosis pathogenesis. Implications for diagnosis and therapy. Trends Mol Med 2001;7:115-121.

23. Boven LA, Van Meurs M, Van Zwam M, et al. Myelin-laden macrophages are anti-inflammatory, consistent with foam cells in multiple sclerosis. Brain 2006;129:517-526.
24. Mikita J, Deloire MS, Canron MH, et al. Prediction of macrophage phenotypes in inflammatory CNS lesions of relapsing EAE disease by MRI with iron nanoparticles. Fifth forum of European Neuroscience Vienna-Austria; July 8-12 2006. A096.13 (abstract).

25. Heyn C, Ronald JA, Mackenzie LT, et al. In vivo magnetic resonance imaging of single cells in mouse brain with optical validation. Magn Reson Med 2006;55:23-29.

26. Slotkin JR, Cahill KS, Tharin SA, Shapiro EM. Cellular magnetic resonance imaging: Nanometer and micrometer size particles for noninvasive cell localization. Neurotherapeutics 4:428-433.

27. Crane IJ, Forrester JV. Th1 and Th2 lymphocytes in autoimmune disease. Crit Rev Immunol 2005;25:75-102.

28. Deloire MS, Touil T, Brochet B, Dousset V, Caille JM, Petry KG. Macrophage brain infiltration in experimental autoimmune encephalomyelitis is not completely compromised by suppressed $\mathrm{T}$ cell invasion: in vivo magnetic resonance imaging illustration in effective anti-VLA-4 antibody treatment. Mult Scler 2004;10:540 548

29. von Adrian UH, Engelhardt B. Alpha 4 integrins as therapeutic targets in autoimmune disease. $N$ Engl J Med 2003;348:68-72.

30. Kappos L, Antel J, Comi G, et al. FTY720 D2201 Study Group. Oral fingolimod (FTY720) for relapsing multiple sclerosis. $N$ Engl J Med 2006;355:1124-1140.

31. Rausch M, Hiestand P, Foster CA, Baumann DR, Cannet C, Rudin M. Predictability of FTY720 efficacy in experimental autoimmune encephalomyelitis by in vivo macrophage tracking: clinical implications for ultrasmall superparamagnetic iron oxide-enhanced magnetic resonance imaging. J Magn Reson Imaging 2004;20:1624.

32. Vollmer T, Key L, Durkalski V, et al. Oral simvastatin treatment in relapsing-remitting multiple sclerosis. Lancet 2004;363:16071608.

33. Toft-Hansen H, Buist R, Sun XJ, Schellenberg A, Peeling J, Owens T. Metalloproteinases control brain inflammation induced by Pertussis toxin in mice overexpressing the chemokine CCL2 in the central nervous system. J Immunol 2006;177:7242-7249.

34. Berger C, Hiestand P, Kindler-Baumann D, Rudin M, Rausch M. Analysis of lesion development during acute inflammation and remission in a rat model of experimental autoimmune encephalomyelitis by visualization of macrophage infiltration, demyelination and blood-brain barrier damage. NMR Biomed 2006;19:101-107.

35. Brochet B, Deloire MS, Touil T, et al. Early macrophage MRI of inflammatory lesions predicts lesion severity and disease development in relapsing EAE. Neuroimage 2006;32:266-274.

36. Raivich G, Bohatschek M, Kloss CU, Werner A, Jones LL, Kreutzberg GW. Neuroglial activation repertoire in the injured brain: graded response, molecular mechanisms and cues to physiological function. Brain Res Brain Res Rev 1999;30:77-105.

37. Dirnagl U, Simon RP, Hallenbeck JM. Ischemic tolerance and endogenous neuroprotection. Trends Neurosci 2003;26:248-254.

38. Schilling M, Besselmann M, Leonhard C, Mueller M, Ringelstein EB, Kiefer R. Microglial activation precedes and predominates over macrophage infiltration in transient focal cerebral ischemia: a study in green fluorescent protein transgenic bone marrow chimeric mice. Exp Neurol 2003;183:25-33.

39. Tanaka R, Komine-Kobayashi M, Mochizuki H, et al. Migration of enhanced green fluorescent protein expressing bone marrow-derived microglia/macrophage into the mouse brain following permanent focal ischemia. Neuroscience 2003;117:531-539.

40. Rausch M, Sauter A, Fröhlich J, Neubacher U, Radu EW, Rudin M. Dynamic patterns of USPIO enhancement can be observed in macrophages after ischemic brain damage. Magn Reson Med 2001; 46:1018-1022.

41. Rausch M, Baumann D, Neubacher U, Rudin M. In-vivo visualization of phagocytotic cells in rat brains after transient ischemia by USPIO. NMR Biomed 2002;15:278-283.

42. Saleh A, Wiedermann D, Schroeter M, Jonkmanns C, Jander S, Hoehn M. Central nervous system inflammatory response after cerebral infarction as detected by magnetic resonance imaging. NMR Biomed 2004;17:163-169.

43. Schroeter M, Saleh A, Wiedermann D, Hoehn M, Jander S. Histochemical detection of ultrasmall superparamagnetic iron oxide 
(USPIO) contrast medium uptake in experimental brain ischemia. Magn Reson Med 2004;52:403-406.

44. Kleinschnitz C, Bendszus M, Frank M, Solymosi L, Toyka KV, Stoll G. In vivo monitoring of macrophage infiltration in experimental ischemic brain lesions by magnetic resonance imaging. J Cereb Blood Flow Metab 2003;23:1356-1361.

45. Wiart M, Davoust N, Pialat JB, et al. MRI monitoring of neuroinflammation in mouse focal ischemia. Stroke 2007;38:131-137.

46. Saleh A, Schroeter M, Jonkmanns C, Hartung HP, Mödder U, Jander S. In vivo MRI of brain inflammation in human ischaemic stroke. Brain 2004;127:1670-1677.

47. Nighoghossian N, Wiart M, Cakmak S, et al. Inflammatory response after ischemic stroke: a USPIO-enhanced MRI study in patients. Stroke 2007;38:303-307.

48. Stoll G, Jander S, Schroeter M. Cytokines in CNS disorders: neurotoxicity versus neuroprotection. J Neural Transm Suppl 2000;59:81-89.

49. Jander S, Schroeter M, Saleh A. Imaging inflammation in acute brain ischemia. Stroke 2007;38:642-645.

50. Gerhard A, Schwarz J, Myers R, Wise R, Banati RB. Evolution of microglial activation in patients after ischemic stroke: a [11c](r)-pk11195 PET study. Neuroimage 2005;24:591-595.

51. Zimmer C, Weissleder R, Poss K, Bogdanova A, Wright SC Jr, Enochs WS. MR imaging of phagocytosis in experimental gliomas. Radiology 1995; 197:533-538.

52. Varallyay P, Nesbit G, Muldoon LL, et al. Comparison of two superparamagnetic viral-sized iron oxide particles ferumoxides and ferumoxtran-10 with a gadolinium chelate in imaging intracranial tumors. AJNR Am J Neuroradiol 2002;23:510-519.

53. Neuwelt EA, Varallyay P, Bago AG, Muldoon LL, Nesbit G, Nixon R. Imaging of iron oxide nanoparticles by MR and light microscopy in patients with malignant brain tumours. Neuropathol Appl Neurobiol 2004;30:456-471.

54. Murillo TP, Sandquist C, Jacobs PM, Nesbit G, Manninger S, Neuwelt EA. Imaging brain tumors with ferumoxtran-10, a nanoparticle magnetic resonance contrast agent. Therapy 2005;2(6): 871-882.

55. Taschner CA, Wetzel SG, Tolnay M, Froehlich J,Merlo A, Radue EW. Characteristics of ultrasmall superparamagnetic iron oxides in patients with brain tumors. AJR 2005;185:1477-1486. 